\title{
Theoretical and experimental validation of a simple method to reproduce representative DEF-prone conditions in laboratory
}

\author{
Renaud-Pierre MARTIN, François TOUTLEMONDE \\ IFSTTAR - Université Paris Est - Bridges and Structures Department \\ Tel: +33140435340 \\ Fax: +33140435343 \\ renaud-pierre.martin@,ifsttar.fr \\ www.ifsttar.fr
}

\begin{abstract}
Delayed Ettringite Formation (DEF) is a possible autogenous expansive reaction of concrete. It can affect materials that have experienced temperatures higher than about $65^{\circ} \mathrm{C}$ at early age. This temperature increase can be the result of the cement heat of hydration in massive structures where the heat transfers are particularly low. To understand the effects of DEF, it is necessary to be able to reproduce thermal DEF-prone conditions in laboratory. This paper proposes a method developed during an extensive experimental programme that aimed at studying the mechanical effects of DEF on structures built with different concretes. The objective was to design a single heat treatment profile to generate homogeneous and similar temperature fields in specimens of various geometries, concrete constituents and mix proportions. It has been demonstrated that quasi-adiabatic conditions were to be realized at early age, whatever the samples size, to simulate the curing conditions in massive structures. The experimental method developed is described in details: the design of the temperature profile, the experimental device and its operation are presented. Both a numeric and an experimental validation are proposed. They emphasize the good accuracy of the process and demonstrate the possibility to trigger similar DEF potential expansions due to the thermal history for all the specimens of the programme.
\end{abstract}

Keywords: Delayed Ettringite formation (DEF); concrete; heat treatment; laboratory test

\section{Introduction}

Delayed Ettringite Formation (DEF) is an autogenous reaction that can affect concrete structures. It concerns materials that have experienced temperatures higher than $60^{\circ} \mathrm{C}$ to $70^{\circ} \mathrm{C}$ (Scrivener et al. 1999 , Taylor et al. 2001, Ramlochan et al. 2003). Due to the cement heat of hydration and low heat transfers, such high temperatures can be measured after the casting of massive structures, which are 
thus particularly prone to DEF (Divet \& Pavoine 2002). The reaction leads to material swelling and mainly implies cracking and decrease of the mechanical properties of the affected materials. As a consequence it gives serious concern regarding the structural integrity and the serviceability of the affected structures. In this context, the managers of civil engineering structures need tools to re-assess the constructions and to predict their mechanical evolution (Baghdadi et al. 2008a, Seignol et al. 2009). This implies an accurate understanding of the effects of DEF.

During the last two decades, numerous theoretical and experimental studies have been performed. However they have mostly dealt with the consequences of the reaction at the material scale (e.g. Heinz \& Ludwig 1987, Fu \& Beaudoin 1996, Famy et al. 2001, Brunetaud et al. 2007). If the corresponding results are useful to analyse the basic features of DEF, they do not take into account all parameters necessary to explain the mechanical behaviour of the affected structures such as stresses, restrained deformations or moisture gradients. The work presented in this paper was part of an experimental programme that aimed at providing data concerning the mechanical effects of DEF on structures (Martin et al. 2008). Thus, in this study, specimens of various dimensions were used: either small cylinders to collect data consistent with the results of the literature or large beams to perform experiments representative of the field cases in terms of moisture and stress gradients.

However, Brunetaud et al. (2007) followed by Baghdadi et al. (2008-b) have studied the quantitative influence of temperature history at early age: they have emphasized the critical effect of this parameter on DEF potential. To correlate and precisely model the results of different experimental tests, it is thus essential to control the temperature history applied to the specimens. To re-create DEF conditions in laboratory, the specimens are systematically heated after casting, basically with a heating phase, a constant temperature plateau and a cooling phase. Most of the studies related in the literature consider a constant heating rate. If this technique seems to be well adapted for small specimens, it may become less efficient for more massive elements where the temperature in the core may not be easily controlled due to the competition between the thermoactivated heat of hydration and the heat transfer processes. 
This paper describes the development and the validation of a single thermal process able to generate a similar DEF potential induced by the temperature history for specimens of various geometries, concrete constituents and mix proportions (Martin 2010): it is thus aimed to take into account both the effect of heat of hydration (which depends on the concrete mix considered) and of geometry (which may induce a delay in temperature control in the core of massive elements). Firstly the design of a heat treatment simulating the curing conditions of a massive concrete structure at early age and specifically adapted for a given concrete mix is described. Prior to any experimental test, numerical modelling was used to theoretically validate the process of temperature control. An experimental validation was then performed. The results of the two approaches are systematically compared to check their consistency. In particular, the homogeneity of the temperature field in the specimens, the scale effects between small and massive elements and the influence of the concrete mix proportions are studied. Finally, the repeatability and the scattering of the process is evaluated in terms of DEF potential expansion.

\section{Heat treatment process}

\subsection{Objective of the study and experimental programme}

In this study, specimens of different geometries were considered. First of all, tests should be performed on small cylinders ( $0.11 \mathrm{~m}$ in diameter, $0.22 \mathrm{~m}$ in height) to evaluate the basic expansion mechanisms at the material scale and quantify the mechanical properties evolution. Secondly, beams $\left(0.25 \times 0.50 \times 3.00 \mathrm{~m}^{3}\right)$ had to be used to assess the structural effects of the pathology.

Three different concrete mixes were to be considered. The mix R1 is made of siliceous sand and aggregates and cement with a high aluminates content: its hydration is very exothermic. This material is thus considered to be strongly prone to DEF. The mix R2 contains the same cement with siliceous sand but includes limestone aggregates reactive regarding Alkali Aggregate Reaction (AAR). This mix is expected to develop combined DEF and AAR processes. Finally, the mix NR has the same sand and aggregates mix-proportions as R1 but its cement has a low aluminates content and is thus significantly less exothermic: it is expected to be less DEF-reactive than R1. Figure 1 illustrates the temperature evolution 
during the hydration of the three concrete mixes in adiabatic conditions according to (Boulay et al. 2010). R1 and R2 have a very similar behaviour. The differences measured can be explained by the higher specific heat of the limestone aggregate corresponding to a higher thermal inertia of the concrete R2. The significant difference between R1 and NR is the consequence of the change of cement.

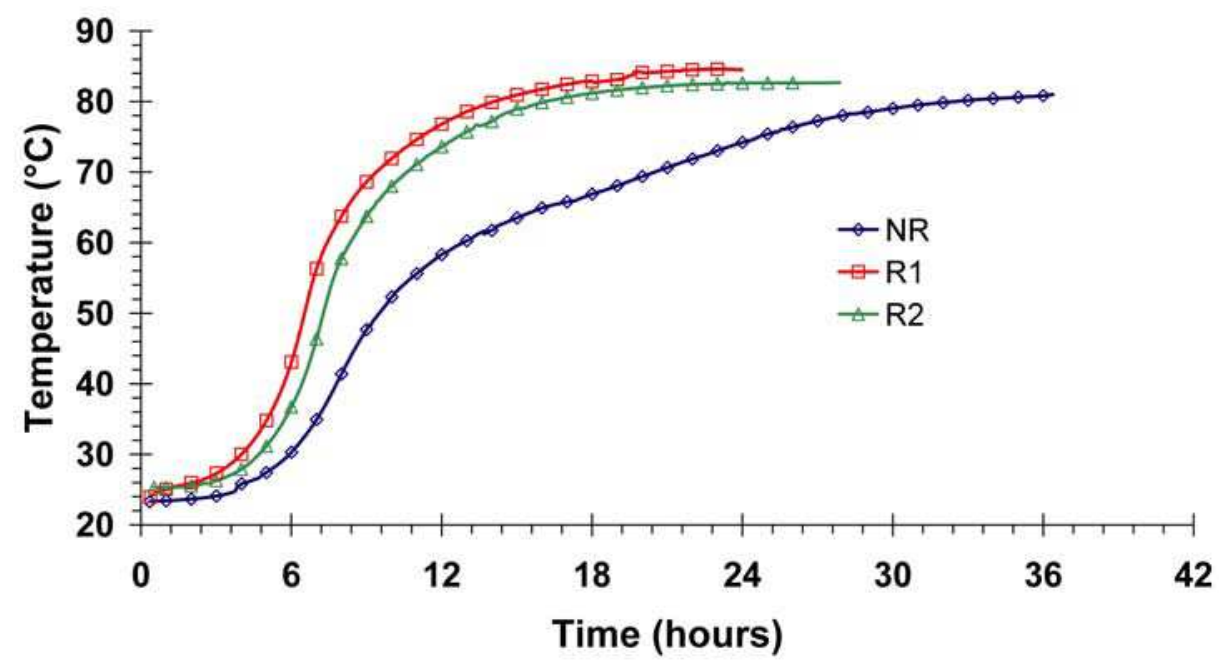

Fig 1. Temperature evolution during hydration in adiabatic conditions for the three concrete mixes

In the experiments finally carried out (Martin et al. 2008), three beams were cast with the R1 mix (they are named R1.1, R1.2 and R1.3 in the following), two beams were cast with the R2 mix (R2.1 and R2.2) and one beam was cast wit the NR mix (NR.1). Due to the capacity of the mixer used and to the available space in the heat treatment device, the casting of the specimens had to be performed at different times with two to three batches at each time. In particular, all the beams had to be cast one by one and separately of all the cylinders.

In order to compare the results of these different tests and eliminate the possible variability of DEF-expansion potential due to imprecise control of early-age thermal history, it has turned out necessary to generate similar temperature histories in all the specimens. As a consequence, the aim of the work detailed in this paper was to design a single heat treatment (also called thermal treatment or curing process in this paper) able to generate a similar and homogeneous temperature field for all specimens, whatever the geometry of the concrete element, its composition or the batch considered. As specimens had to be cast at different periods, the process had to be repeatable as well. 
Technical development and optimization of the temperature control process was based both on empirical and theoretical considerations, detailed here because of their general interest and applicability.

\subsection{Technical requirements}

Experimentally, the heat treatment has to be started two hours after the beginning of the casting of the specimens to allow fresh concrete handling in laboratory. The thermal history has been idealized in three steps: a heating phase, a constant temperature plateau and a cooling phase. This schematic shape has been used to simplify the process and to ensure its repeatability during the whole experimental programme.

The constant temperature step was considered to be the most critical phase regarding the DEF potential. Therefore, it was decided that the temperature differences during this step must not exceed $\pm 1^{\circ} \mathrm{C}$, whatever the concrete mix, the geometry or the casting considered. The relevance of this criteria regarding the DEF potential can be assessed thanks to the model developed in (Baghdadi et al 2008-b) and given by the equation (1) where $\varepsilon_{\infty}$ is the estimated DEF potential expansion, $\alpha$ is a constant corresponding to the concrete mix, $\mathrm{T}(\mathrm{t})$ is the temperature at time $\mathrm{t}, \mathrm{t}_{\mathrm{m}}$ the mature time of concrete, $\mathrm{T}_{0}$ is a threshold temperature below which no DEF risk is considered, $\mathrm{E}_{\mathrm{a}}$ is the specific activation energy of the reaction and $\mathrm{R}$ is the gas constant. This model is based on the assumption that the destabilization of ettringite necessary for DEF to occur is thermo-activated as it has been discussed by (Pourchez et al. 2006). It is stated that this process obeys an Arrhenius-type law once the temperature is high enough to make ettringite soluble. An integral function is used to take into account both the curing temperature and the period during which it is maintained.

$$
\varepsilon_{\infty}=\alpha \cdot \int_{0}^{t_{m}} \Phi(t) \cdot d t \text { where } \Phi(t)=\left\{\begin{array}{l}
0 \text { if } T(t) \leq T_{0} \\
\exp \left(-\frac{E_{a}}{R} \cdot \frac{1}{T(t)-T_{0}}\right) \text { else }
\end{array}\right.
$$

(Martin et al. 2012) have described a methodology to assess the different parameters of Baghdadi's law for a concrete similar to R1: values are estimated by fitting the predictions to the experimental expansions of concrete elements submitted to heat treatments of various maximum temperatures and durations. 
Values of $\alpha, \mathrm{E}_{\mathrm{a}}$ and $\mathrm{T}_{0}$ representative of the $\mathrm{R} 1 \mathrm{mix}$ have been proposed in (Martin 2010). They are equal to $6.48 .10^{-4} \mathrm{~h}^{-1}, 435 \mathrm{~J}^{\mathrm{mol}}{ }^{-1}$ and $43.3^{\circ} \mathrm{C}$ respectively. Considering a constant temperature step of $80^{\circ} \mathrm{C}$ during 72 hours, and before and after this plateau, heating and cooling at rates of $5^{\circ} \mathrm{C} / \mathrm{h}$, the precision criterion of $\pm 1^{\circ} \mathrm{C}$ imposed during the plateau corresponds according to equation (1) to absolute differences of $\pm 0.05 \%$ for an estimated mean DEF potential of $1.20 \%$ (relative scatter of $\pm 4 \%$ ). Such a scatter estimation seems to be consistent when compared to the multiple sources of uncertainties of such experiments (e.g. precision of the measurement device, operators, storage temperature variations). Yet it is emphasized that a precise control of temperature history during this early age curing is of outmost significance on the control of the DEF development.

Moreover, since the curing process aims to simulate the maturation conditions existing in the core of a massive structure at early age, the thermal curing process should be performed under water to favour high internal humidity: indeed the internal relative humidity of concrete at early age is maintained at high levels for many days as shown in (Grasley et al. 2006). This high humidity favours thermal inertia, which shall be accounted for in developing the technology of the thermal curing process.

\subsection{Description of the thermal curing process}

The design of the temperature profile of the heat treatment has been described in details in (Martin \& Toutlemonde 2010). It consists in curing the concrete specimens right after casting in water whose temperature is precisely controlled. The most complicated part of the process corresponds to the heating phase: during this step, there is a competition between the heat generated by the thermoactivated hydration of cement and the heat transfers. Thus it implies a size effect: for small specimens like cylinders, the volume is small compared to the exchange surface. As a consequence, the temperature of the core of the element is quite easily controlled by the curing temperature and the heat of hydration represents a minor contribution to the temperature evolution. For bigger specimens like the beams considered in this work, the curing temperature has a much smaller effect at the core. Thus, the heat of hydration strongly drives the inner temperature in this case. 
Figure 2 illustrates this phenomenon for elements submitted to a constant heating (which is generally the case in the studies of small specimens reported in the literature) at a rate of $2.5^{\circ} \mathrm{C} / \mathrm{h}$. Using the TEXO module of the CESAR-LCPC finite element code (Humbert et al. 2005) the temperature evolutions in a cylinder and a beam were simulated. Due to the thermoactivation of cement hydration and the lower heat transfers within the massive beam, significant temperature differences are estimated between the cores of the two specimens, with a maximum gap of $13^{\circ} \mathrm{C}$. Using equation (1), the DEF potentials at the core of the beam and of the cylinder are estimated at $0.11 \%$ and $0.14 \%$ respectively corresponding to a relative variation of about $21 \%$. Similarly, the calculated DEF potential of a point located on the side face of the beam is equal to $0.13 \%$ corresponding to a relative discrepancy of about $18 \%$ with the potential at the core. Thus, if the phase of temperature increase is only controlled by an external constant heating rate of the fluid surrounding the specimens, it would lead to different DEF potentials for each specimen shape. Moreover, it would generate an inhomogeneous DEF potential field within the beam leading to internal cracking because of the differential expansions. Finally, a higher temperature maximum within the beam would imply a risk of thermal cracking at the surface during the heat treatment. Such a thermal curing procedure is thus not relevant regarding our technical requirements aiming at providing homogenous processes in the specimens to facilitate the interpretation of the experimental results.

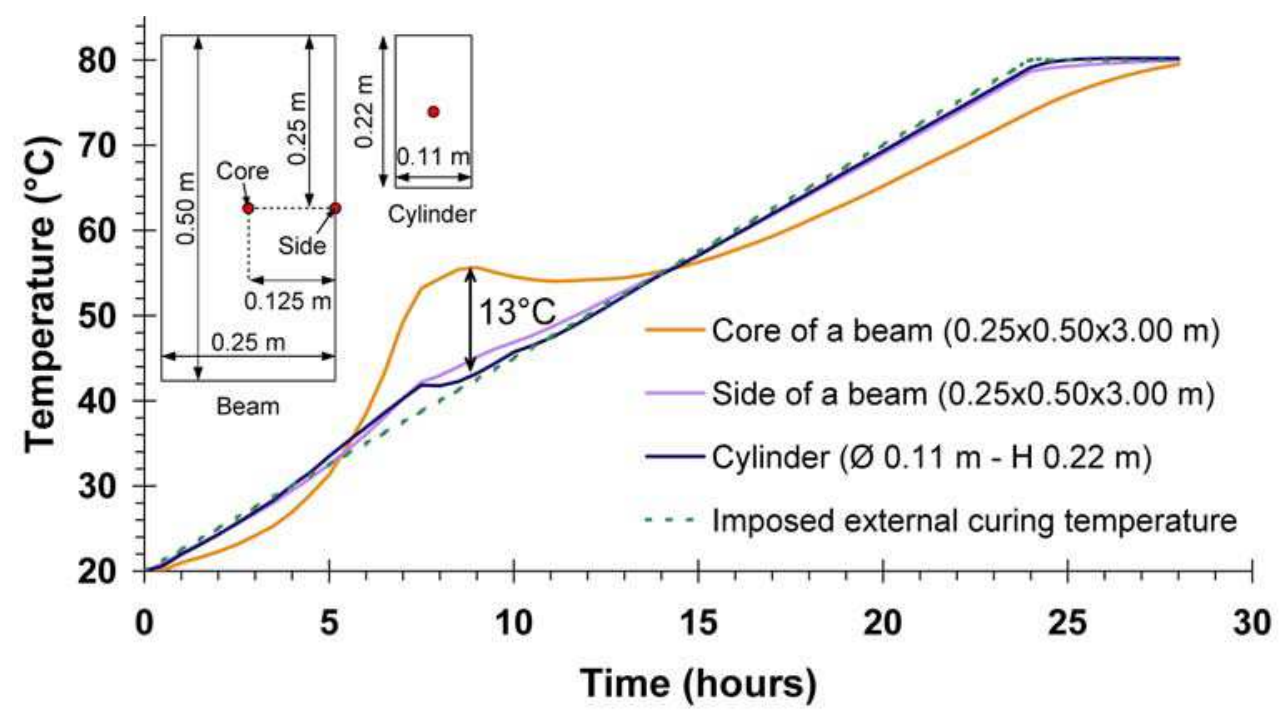

Fig. 2 Simulation of the temperature evolution in concrete elements submitted to a constant heating rate $\left(2.5^{\circ} \mathrm{C} / \mathrm{h}\right)$ 
To avoid such size effects, the constant heating phase was replaced by simulated adiabatic conditions: the heating rates were estimated thanks to the results of the adiabatic test of the R1 mix (see Figure 1). Such an approach lowers the temperature difference between the core and the surface of massive specimens since the temperature boundary condition imposed at the surface is in this case driven by the heat generated by the hydration of cement. A simulation of the temperature evolution during the heating phase leads to an estimation of a DEF potential expansion of about $0.27 \%$ whatever the geometry and the location within the specimen considered.

The constant temperature step corresponds to a temperature of $81^{\circ} \mathrm{C}$. This value is imposed by the temperature evolution during hydration in adiabatic conditions and is a consequence of the technique chosen for the heating phase. The plateau has a duration of 72 hours which is supposed to be representative of the curing conditions of massive concrete structures (Taylor et al. 2001, Divet 2002, Martin $\&$ Toutlemonde 2010). Finally, the specimens are cooled down at a constant rate of $-1^{\circ} \mathrm{C} / \mathrm{h}$ which is assumed to be slow enough to avoid thermal cracking. The global duration of the process is about 6 days. Figure 3 represents the theoretical temperature evolution during the heat treatment.

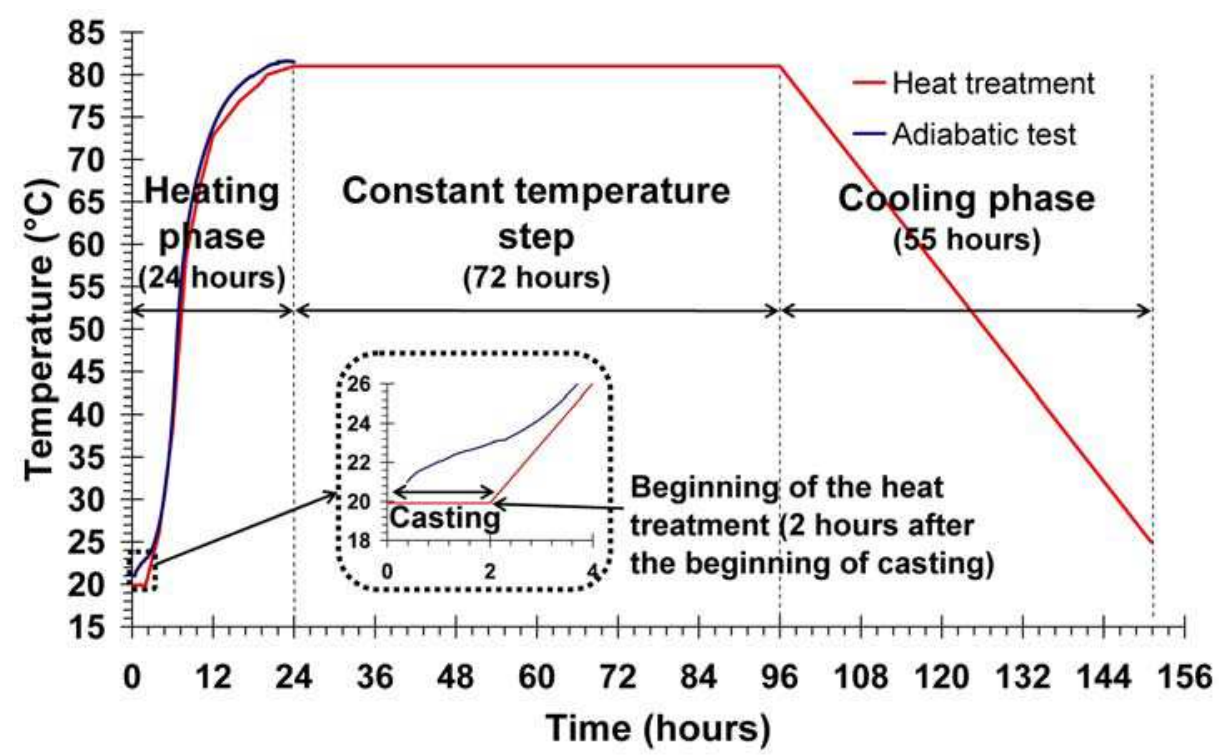

Fig. 3 Temperature evolution during the heat treatment compared to the results of an adiabatic test 


\subsection{Experimental device}

The heat treatment device specifically designed for this work is represented in figure 4. It consists in a tank $\left(3.50 \times 0.85 \times 0.75 \mathrm{~m}^{3}\right)$ filled with water whose temperature can be set thanks to an electrical heater $(24 \mathrm{~kW})$ controlled by a programmable automat: a constant monitoring of the temperature at entrance and exit of the storage volume (respectively $\mathrm{T}_{\text {in }}$ and $\mathrm{T}_{\text {out }}$ in figure 4 ) allows a computer to set the output power of the heater in order to regulate the temperature of the curing water. The device can contain one beam at most. To ensure homogeneity of the temperature, a water circulation is provided thanks to a pump. No cooling system is incorporated in the device: cooling is obtained by heat exchange with the surrounding atmosphere and / or injection of cold water in the tank. The maximum heating rate is equal to $10^{\circ} \mathrm{C} / \mathrm{h}$.
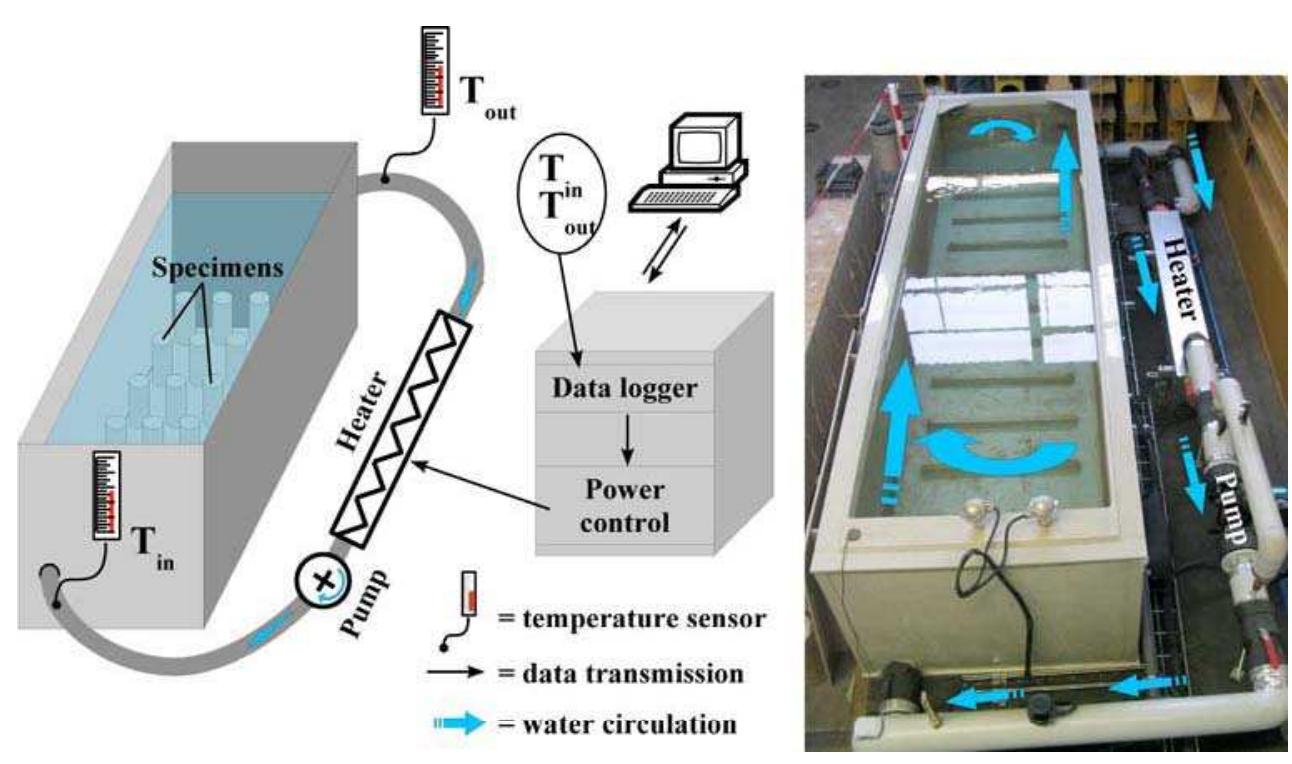

Fig. 4 Heat treatment device

Figure 5 illustrates an experimental temperature profile measured in the tank during a heat treatment. It was checked that the required temperature signal is reached with an accuracy of $\pm 0.5^{\circ} \mathrm{C}$ in steady state. Moreover, the temperature deviations in the tank have been checked to be lower than $\pm 1^{\circ} \mathrm{C}$ during the phase of maximum heating. The process is repeatable with an accuracy of $\pm 0.5^{\circ} \mathrm{C}$ regarding the mean water temperature in the tank. To quantify the repeatability of the thermal process in terms of potential expansion provided, equation (1) has been used. Calculations have been performed for the 12 successive heat 
treatments effectively carried out during the experimental programme. Using the parameters given in section 2.2, the mean value of the potential expansion theoretically provided (i.e. if the internal temperature of the specimen perfectly followed the temperature of the bath) is $1.582 \%$ with a standard deviation of $0.002 \%$.

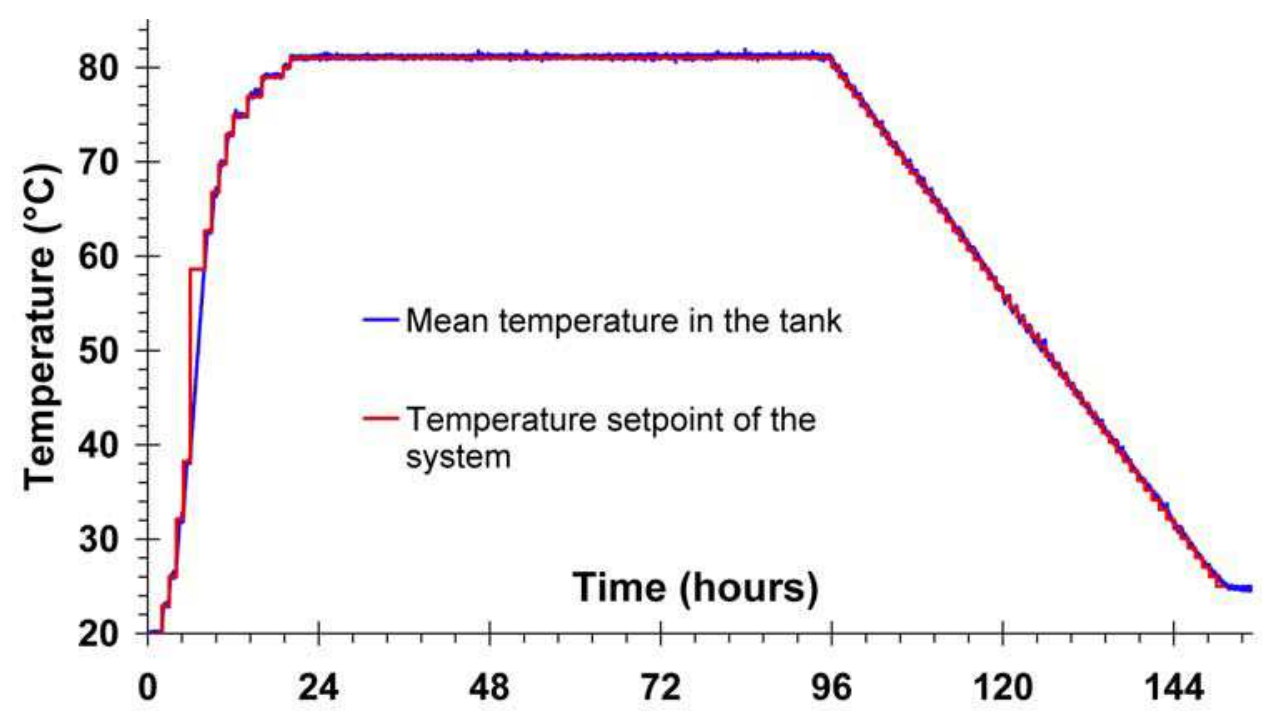

Fig. 5 Experimental temperature profile in the tank during a heat treatment

\section{Effectiveness control of the heat treatment process}

\subsection{Assessment methodology}

\subsubsection{Numerical modelling}

To assess the relevance of the heat treatment process prior to the casting of the specimens, numerical simulations of the heat treatment were performed using the TEXO module of the CESAR-LCPC finite element code as it is performed in (Tailhan et al. 2010) for example. For this model, the main input data concerning the material characteristics is the heat evolution during hydration of the concrete in adiabatic conditions (see figure 1). Table 1 synthesizes the characteristics of the models. The specific heat of the material and its thermal conductivity properties were chosen to be representative of an ordinary concrete (CESAR-LCPC 2001). 
The heat transfer coefficient was chosen to be representative of a storage in water (Lienhard \& Lienhard 2008). This choice was confirmed as reasonable and not critical after a sensitivity analysis which emphasized that an increase of a factor 10 of the heat transfer coefficient implies minor variations in the calculations corresponding to the present study.

These calculations have given a first validation a the thermal control process since all technical requirements were checked to be satisfied. The modelling results were then compared to the experimental data to check the relevance of our approach (see section 3.2).

Table 1. Input data of the different numerical simulations

\begin{tabular}{|l|l|l|}
\hline \multicolumn{2}{|l|}{ Beam model } & $2 \mathrm{D}$ \\
\hline \multicolumn{2}{|l|}{ Cylinder model } & $2 \mathrm{D}$, axisymmetric \\
\hline Heat transfer coefficient at formwork/water interface & $100 \mathrm{~W} /\left(\mathrm{m}^{2} .{ }^{\circ} \mathrm{C}\right)$ \\
\hline \multirow{3}{*}{ Thermal conductivity } & $\mathrm{x}$ direction & $1.67 \mathrm{~W} /\left(\mathrm{m}^{2} .{ }^{\circ} \mathrm{C}\right)$ \\
\cline { 2 - 3 } & $\mathrm{y}$ direction & $1.67 \mathrm{~W} /\left(\mathrm{m}^{2} .{ }^{\circ} \mathrm{C}\right)$ \\
\cline { 2 - 3 } & $\mathrm{x} / \mathrm{y}$ & $0($ isotropic $)$ \\
\hline Specific heat of hardened concrete $\mathrm{C}_{\mathrm{th}}$ & $2400 \mathrm{~kJ} /\left(\mathrm{m}^{3} . \mathrm{K}\right)$ \\
\hline
\end{tabular}

\subsubsection{Experimental measurements}

To assess experimentally the thermal response of the concrete specimens during the heat treatment, temperature sensors (accuracy of $\pm 0.3^{\circ} \mathrm{C}$ at $25^{\circ} \mathrm{C}$ ) were placed in the formworks before fresh concrete was placed. Temperature data were recorded every minute in the centre of a cylinder and in five different points of each beam corresponding to depths of $0.04 \mathrm{~m}, 0.08 \mathrm{~m}, 0.17 \mathrm{~m}, 0.27 \mathrm{~m}$ and $0.37 \mathrm{~m}$ from the upper face during the whole heat treatment.

\subsection{Results}

\subsubsection{Thermal response of a beam}

The results presented in this section correspond to the beam R1.1. This specimen is made of the concrete mix R1 for which the heat treatment was designed. Figure 6 illustrates the temperature evolution in the beam during the heating phase during 
which the heat of hydration has the most critical influence. The comparison of the experimental monitoring and of the results of the calculation shows reasonable consistency. Thus, prior to any experimental test, the numerical simulation has shown to provide valuable elements to validate the design of the heat treatment process. In particular, almost no overrun of the imposed external temperature is observed in the specimen at the beginning of the constant temperature step: this demonstrates the efficiency of a heating phase determined by the heat evolution of the material in adiabatic conditions to avoid loss of temperature rise control as illustrated by figure 2 .

However, some significant differences can be observed between the temperature in the beam and the curing temperature, especially between 8 and 12 hours after the beginning of the test. This can be explained by the fact that adiabatic conditions are not perfectly reproduced by the imposed curing temperature. As a consequence, during this period, the heat generated by the thermoactivated hydration is high enough to induce a significant internal temperature elevation. This phenomenon remains however limited.

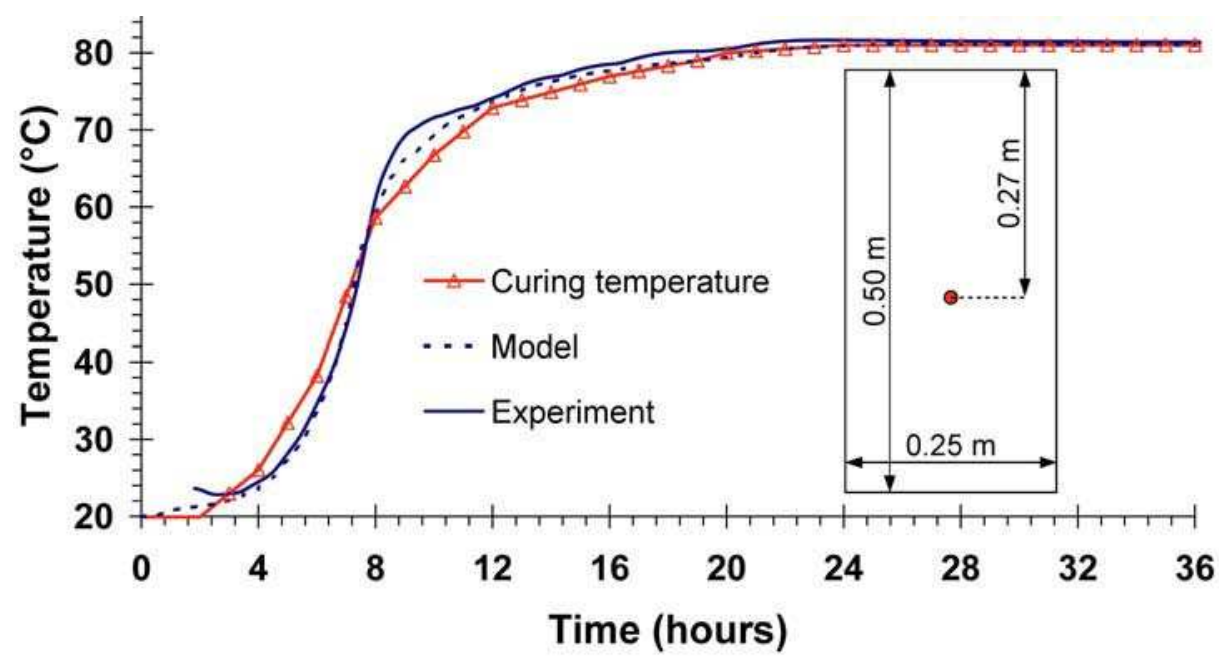

Fig. 6 Temperature evolution at a depth of $0.27 \mathrm{~m}$ in the beam R1.1 during heating

Figure 7 represents the temperature differences between points located at a depth of $0.27 \mathrm{~m}$ and $0.04 \mathrm{~m}$ from the upper face of the beam respectively. Although some differences are noted between the model and the experiment and despite some deviations of the simulation of adiabatic conditions emphasized by figure 6 , experimental results and computation similarly predict limited deviations, mostly during non-steady phases of the thermal curing. This demonstrates the efficiency 
of performing this kind of calculation prior to the realization of such an experimental programme. In particular, it is shown that the temperature differences between the core of the beam and its surface (respective depths of $0.27 \mathrm{~m}$ and $0.04 \mathrm{~m}$ ) remain much smaller than $\pm 1^{\circ} \mathrm{C}$ during the constant temperature step (i.e. between 24 and 96 hours) and thus satisfy the precision criterion defined in section 2.1.

During cooling ( $\mathrm{t}>96$ hours), it can be noted that the calculation overestimates the temperature gradient in the beam. It can be explained by an overestimation of the specific heat of the material in the calculation (which is quite difficult to assess as the composition of the system constantly evolves as noted by (Waller 2000)): it is experimentally easier to cool down the specimen. The temperature gradient is thus smaller.

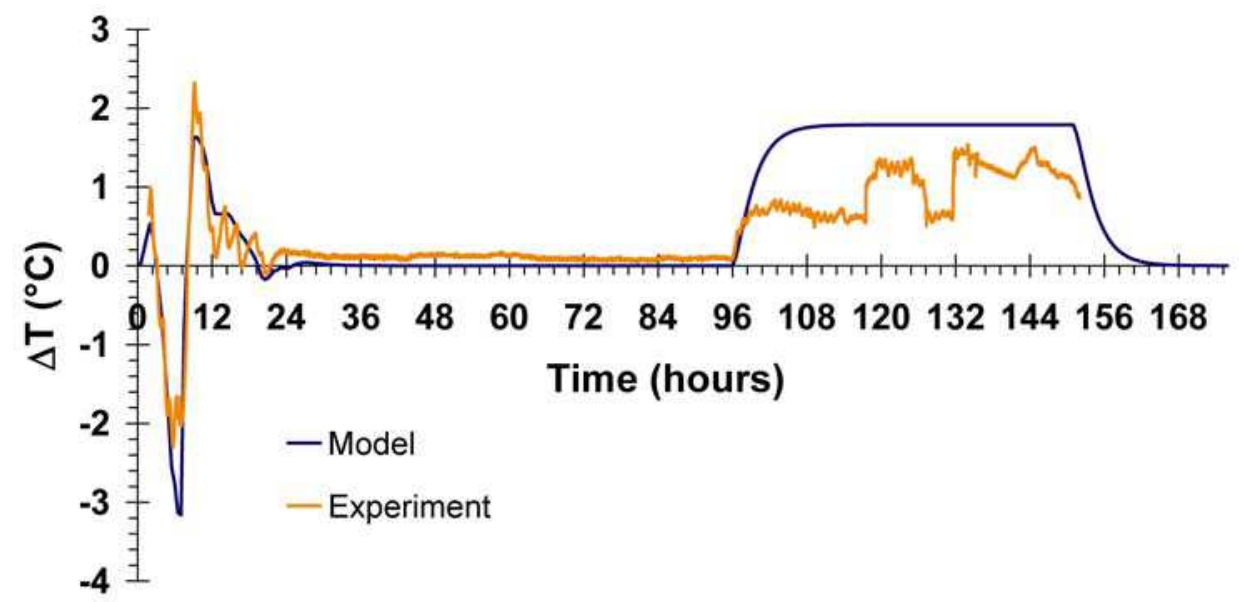

Fig. 7 Evolution of the temperature difference between the core and the surface (respective depths of $0.27 \mathrm{~m}$ and $0.04 \mathrm{~m}$ from the upper face) in beam R1.1

\subsubsection{Effect of the specimens geometry}

Figure 8 illustrates the evolution of the temperature differences between the core of the beam R1.1 (depth of $0.27 \mathrm{~m}$ ) and the core of a cylinder made out of the same concrete mix. These two specimens were cast at two different times. Theoretical and experimental results are in good agreement and emphasize once again that this kind of modelling can be very useful to validate such a process prior to any experimental test.

The temperature difference during the constant temperature plateau (between 24 and 96 hours) is much smaller than $\pm 1^{\circ} \mathrm{C}$. This result validates our process 
regarding the precision criterion defined in section 2.2: the heat treatment is able to apply a similar temperature profile for specimens made of the same mix but of various geometries and thus ensure a similar DEF potential. This validates the use of this single temperature profile for specimens made out with the concrete mix $\mathrm{R} 1$, whatever their geometry.

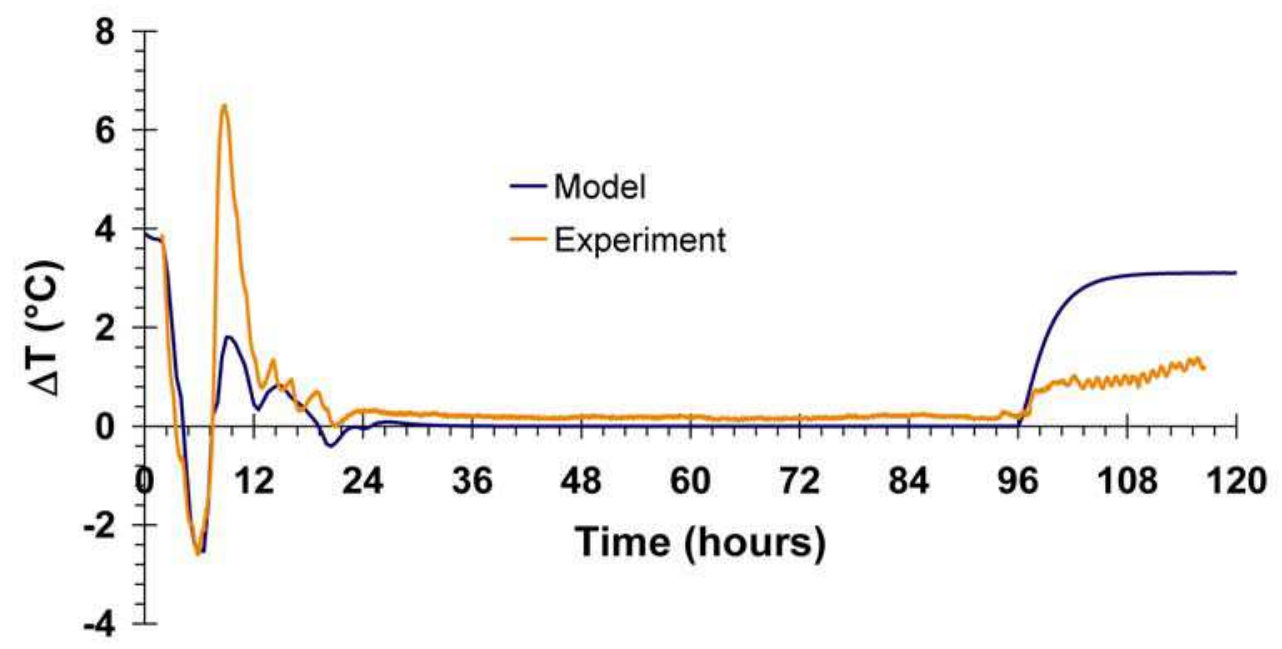

Fig. 8 Evolution of the temperature differences between the core of the beam R1.1 and the centre of a cylinder cast with the same concrete

\subsubsection{Influence of the concrete mix design}

The heating phase of the thermal treatment was designed to fit as good as possible the heat development of the concrete mix R1 in adiabatic conditions. However, three different concrete mixes had to be used in this experimental programme. To limit the differences in the casting processes of the different elements, it was considered as practically preferable, if possible, to apply the same heat treatment to all kind of specimens. Thus, it was necessary to check the influence of the specific exothermic behaviour of each concrete mix on the thermal response of the different specimens in order to verify if only one temperature profile could be used for the different mixes. In the calculation, this corresponds to the change of the input data corresponding to the heat development of the concrete mix in adiabatic conditions.

Figure 9 illustrates the temperature differences between the beams R1.1, NR.1 and $\mathrm{R} 2.1$ at a depth of $0.27 \mathrm{~m}$. Although some differences are observed at the beginning of the test due to the influence of the initial temperature of the 
materials, the general behaviour obtained from the computations is in good agreement with the results of the tests for all cases. As it was expected, the differences predicted by the model for NR.1 are higher than for R2.1: this can be explained by the significant difference of adiabatic thermal behaviour of the mix NR relatively to R1 (see figure 1); on the contrary, since the mix R2 has a similar behaviour to R1, the temperature differences between the beams R1.1 and R2.1 are lower. Experimentally, the temperature differences measured are smaller than the predictions of the models which can be explained by the different initial thermal state and the possibility of an overestimation of the specific heat of the materials as it was noted in section 3.2.1. In any case, the maximum deviations between the different beams remain smaller than $\pm 1^{\circ} \mathrm{C}$ during the constant temperature step and are thus in agreement with the precision criterion, defined in this study.
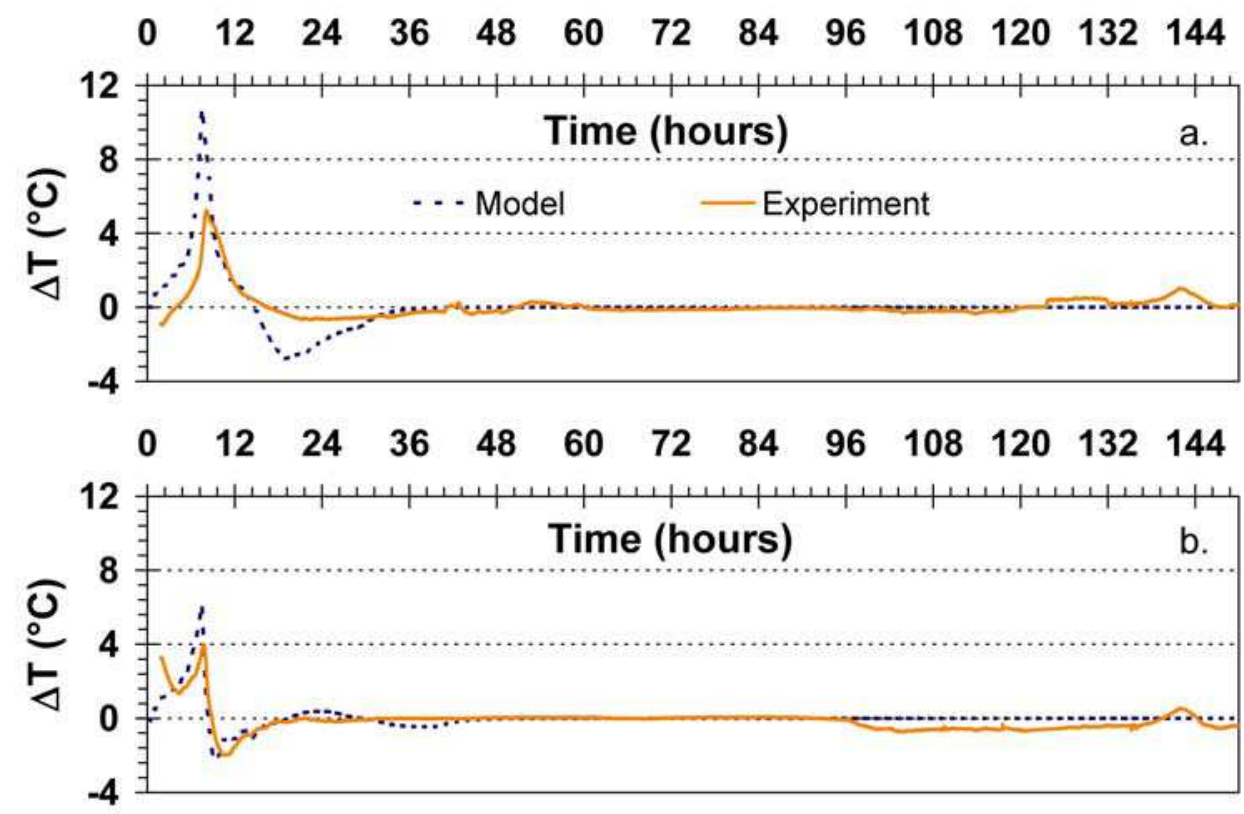

Fig. 9 Temperature difference between the beam R1.1 and the beams NR.1 (a.) and R2.1 (b.) at a depth of $0.27 \mathrm{~m}$ 


\subsection{Repeatability and dispersion of the DEF potential provided}

\subsubsection{Repeatability of the DEF potential in beams made out of the same}

concrete mix

Figure 10 illustrates the evolution of the temperature differences between the beams made of the concrete mixes R1 and R2. The higher temperature differences between two consecutive batches are measured during the 12 first hours of the heat treatment. They mainly remain smaller than $\pm 2^{\circ} \mathrm{C}$ and can be explained by the ambient temperature variations during the casting: as an example, the initial temperatures of the beams R1.1, R1.2 and R1.3 were respectively 23.6, 20.6 and $26.1^{\circ} \mathrm{C}$. These deviations are rapidly homogenised due to the combined effects of curing and heat of hydration. In particular, during the constant temperature step, the differences remain much smaller than $\pm 1^{\circ} \mathrm{C}$ and thus satisfy the precision criterion required for the heat treatment.

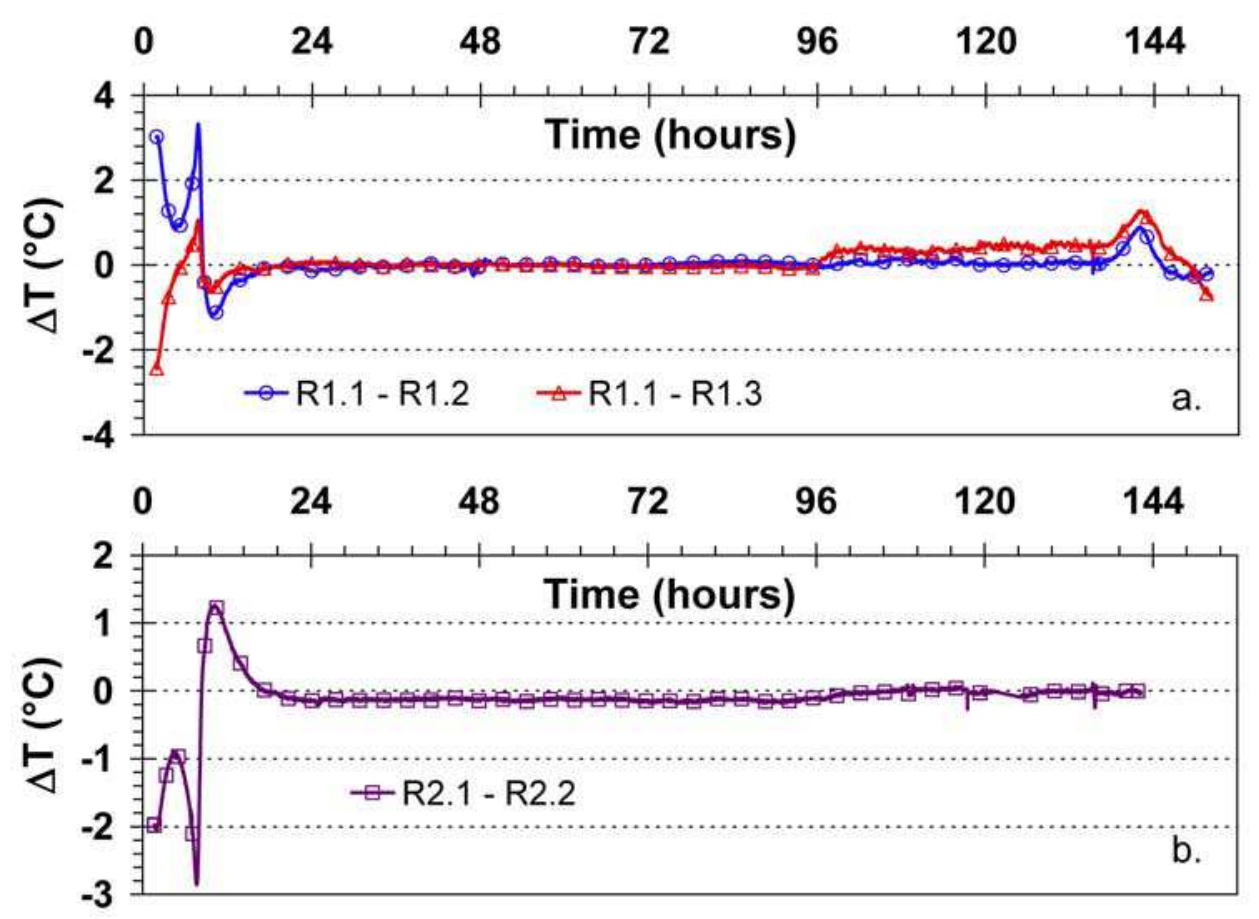

Fig. 10 Experimental temperature differences of beams made out of the R1 (a.) and R2 (b.) concrete mixes at a depth of $0.27 \mathrm{~m}$ 


\subsubsection{DEF potential expansion field in the beams}

Although the heating phase has been designed to minimize the thermal gradients in the specimens, it has been shown in the previous sections that some temperature differences could have appeared due to the imperfection of the simulation of adiabatic conditions, the variation of initial thermal state during casting of the elements and the use of the same heat treatment process for specimens made of different mixes. These temperature deviations have an influence on the DEF potential expansion generated in the materials as shown by (Baghdadi et al. 2008-b).

Figure 11 illustrates the profile of DEF potential expansion after heat treatment estimated thanks to relation (1) using values of $6.48 .10^{-4}$ for $\alpha, 43.3^{\circ} \mathrm{C}$ for $\mathrm{T}_{0}$ and 435, 440 and $895 \mathrm{~J} \mathrm{~mol}^{-1}$ for $\mathrm{E}_{\mathrm{a}}$, for R1, R2 and NR mixes respectively as determined in (Martin 2010).

Due to a failure of the sensor at a depth of $0.17 \mathrm{~m}$ in the beam R2.1, the corresponding information is missing. As it was expected, the deeper the measurement point from the outer surfaces, the higher the potential expansion. This increase is due to the contribution brought by the heat of hydration: the points located deeper in the beam are less influenced by the imposed curing temperature which leads to an increased relative effect of the heat of hydration. However, the standard deviation of the DEF potential for a given concrete mix is extremely limited and respectively equal to $0.004 \%, 0.011 \%$ and $0.012 \%$ for the NR, R1 and R2 mixes. The corresponding mean potential expansions are $0.35 \%$, $1.61 \%$ and $1.60 \%$. The obtained deviation thus corresponds to less than $1.2 \%$ of relative scattering.

The DEF potentials corresponding to the theoretical situation where the temperature of the specimens perfectly followed the imposed curing temperature delivered by the heat treatment device were estimated in the same way with equation (1). Their mean values are equal to $0.34 \%, 1.58 \%$ and $1.56 \%$ for NR, $\mathrm{R} 1$ and R2 respectively. They are compared in table 2 to the estimated DEF potential (according to equation (1)) and to the corresponding highest relative deviation within each beam. In comparison to the theoretical situation of specimens perfectly following the imposed curing temperature, the imperfection of the simulated adiabatic conditions implying a thermoactivation of hydration leads to a relative increase of the potentials of about $3 \%, 2 \%$ and $3 \%$ for the 
specimens made out of the NR, R1 and R2 mixes respectively. These results attest of the ability of the thermal treatment process to generate a repeatable and homogeneous DEF potential field in massive structures made of various concrete mixes while compared to a process with a constant heating rate (see section 2.3). Moreover, the criterion of a scatter of less than $4 \%$ of the DEF potential within the same specimen is satisfied (see table 2).

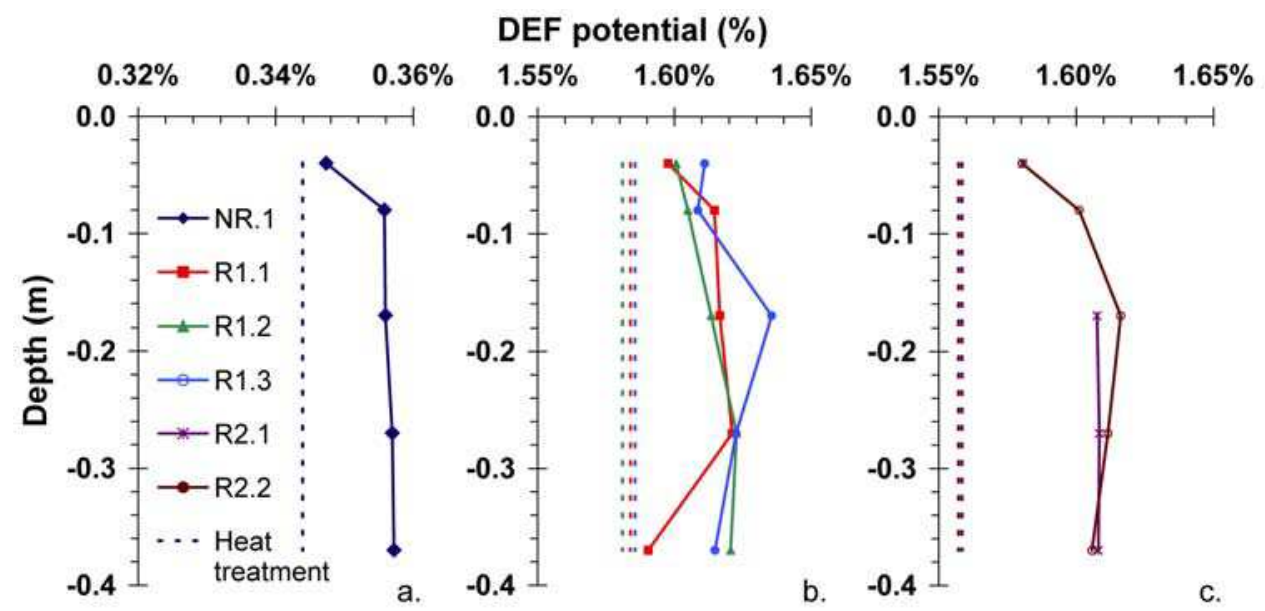

Fig. 11 DEF potential expansion profiles in the beams after heat treatment (a. NR ; b. R1 ; c. R2)

Table 2. DEF potential characteristic values within the beams

\begin{tabular}{|l|l|l|l|l|l|l|}
\hline & NR.1 & R1.1 & R1.2 & R1.3 & R2.1 & R2.2 \\
\hline $\begin{array}{l}\text { Theoretical DEF } \\
\text { potential provided }\end{array}$ & $0.34 \%$ & $1.58 \%$ & $1.58 \%$ & $1.59 \%$ & $1.56 \%$ & $1.56 \%$ \\
\hline $\begin{array}{l}\text { Minimum DEF } \\
\text { potential in the beam }\end{array}$ & $0.35 \%$ & $1.59 \%$ & $1.60 \%$ & $1.61 \%$ & $1.58 \%$ & $1.58 \%$ \\
\hline $\begin{array}{l}\text { Maximum DEF } \\
\text { potential in the beam }\end{array}$ & $0.36 \%$ & $1.62 \%$ & $1.62 \%$ & $1.64 \%$ & $1.61 \%$ & $1.62 \%$ \\
\hline $\begin{array}{l}\text { Maximum relative } \\
\text { deviation in the beam }\end{array}$ & $2.76 \%$ & $1.90 \%$ & $1.36 \%$ & $1.65 \%$ & $1.73 \%$ & $2.22 \%$ \\
\hline
\end{tabular}

\section{Conclusions}

This paper has detailed the development and validation of an original heat treatment process able to generate similar DEF potential expansions in specimens of various geometries and reproduce the same thermal history whatever the 
concrete mix. Indeed, due to the competition between the imposed curing temperature and the heat generated by hydration of cement, significant temperature differences can develop in the specimens if careful optimization of the thermal control is not achieved which can rise problems in terms of comparison of expansive behaviour of the DEF-prone concretes.

The main difficulty turned out to take place during the heating phase of massive specimens: in this phase, the temperature at the surface is mainly driven by the imposed curing temperature whereas the heat of hydration has a major influence in the core. To avoid such effects, the heating phase was controlled to follow the heat generated during the hydration of concrete in adiabatic conditions. This process has been shown to be effective: it allowed to apply a homogeneous temperature field in specimens of various dimensions and avoided that the temperature at the core of the specimen exceeds the curing temperature during the transition between the heating phase and the constant temperature plateau. The scale effects were proved to be negligible: the comparison of the temperature in the core of a massive beam and of a small cylinder have shown differences to be very limited. In particular, the deviations turned out to be much smaller than $\pm 1^{\circ} \mathrm{C}$ during the constant step which is critical regarding the DEF potential expansions.

A robustness study has shown that the same heat treatment could be used for specimens made of various mixes: although temperature deviations were observed (especially during the heating phase), their impact was very limited. In particular, the DEF potential expansion generated in massive beams has turned out very homogeneous which fulfilled the necessary condition to assess accurately the mechanical effects of this pathology on laboratory structures and calibrate chemomechanical models.

\section{Acknowledgments}

The authors are particularly grateful to F.-X. Barin, C. Bazin, J. Billo, M. Estivin, L. Lauvin and J.-C. Renaud (Structures and Bridges department of Paris Est University - Ifsttar) for their involvement during the experimental validation of the heat treatment. D. Siegert (Measurement, monitoring and scientific computations department at Ifsttar) is also gratefully acknowledged for his help during the design and validation of the heat treatment device. Finally, the authors 
would like to thank E. Bourdarot and A. Jeanpierre (Electricité de France) for the financial support and technical partnership in this research programme.

\section{References}

Baghdadi N., Seignol J.-F., Toutlemonde F. (2008-a) Chemo-mechanical model describing the expansion due to internal sulfate attack: numerical simulation. International RILEM Symposium on Concrete Modelling CONMOD’08, Delft, The Netherlands: 291-298.

Baghdadi N., Seignol J.-F., Martin R.-P., Renaud J.-C., Toutlemonde F. (2008-b) Effect of early age thermal history on the expansion due to delayed ettringite formation: experimental study and model calibration. Euro Mediterranean symposium on Advances in Geomaterials AGS'08, Hammamet, Tunisia: 661-666.

Boulay C., André J.-L., Torrenti J.-M. (2010) Draft operating protocol to determine the level of heat released during cement hydration on a concrete specimen placed in a quasi-adiabatic calorimeter designed for concretes (QAB). Bulletin des Laboratoires des Ponts et Chaussées 278: $37-42$.

Brunetaud X., Linder R., Divet L., Duragrin D., Damidot D. (2007) Effect of curing conditions and concrete mix design on the expansion generated by DEF. Materials and Structures 40: 567578.

CESAR-LCPC (2001) Manuel d'exemples - Modélisation du béton au jeune âge. In: CESARLCPC : Documentation utilisateur, version 3.3, vol. 4, $3^{\text {rd }}$ edn. 61 p.

Divet L. (2002) Comment se prémunir des réactions sulfatiques dans les bétons ? Bulletin des Laboratoires des Ponts et Chaussées 240: 87-94.

Divet L. \& Pavoine A. (2002) Delayed Ettringite Formation in massive concrete structures: an account of some studies of degraded bridges. International RILEM TC 186-ISA workshop on Internal Sulfate Attack and Delayed Ettringite Formation, Villars, Switzerland: 98-126.

Famy C., Scrivener K.L., Atkinson A., Brough A.R. (2001) Influence of the storage conditions on the dimensional changes of heat-cured mortars. Cement and Concrete Research 31: 795-803.

Fu Y. \& Beaudoin J.J. (1996) Mechanisms of Delayed Ettringite Formation in Portland cement systems. ACI Materials Journal 93(4): 1-7.

Grasley Z.C., Lange D.A., D’Ambrosia M.D. (2006) Internal relative humidity and drying stress gradients in concrete. Materials and Structures 39: 901-909.

Heinz D. \& Ludwig U. (1987) Mechanisms of secondary ettringite formation in mortars subjected to heat treatment. ACI SP 100: 2059-2071.

Humbert P., Dubouchet A., Fezans G., Remaud D. (2005) CESAR-LCPC, un progiciel de calcul dédié au Génie Civil. Bulletin des Laboratoires des Ponts et Chaussées 256-257: 7-37.

Lienhard J.H. IV and Lienhard J.H. V (2008) A heat transfer textbook, $3^{\text {rd }}$ edn. Phlogiston Press, Cambridge, MA.

Martin R.-P., Siegert D., Toutlemonde F. (2008) Experimental analysis of concrete structures affected by DEF - Influence of moisture and restraint. $3^{\text {rd }}$ International symposium on thermo- 
hydromechanical and chemical couplings in geomaterials and applications GEOPROC'08, Lille, France: 589-596.

Martin R.-P. (2010) Analyse sur structures modèles des effets mécaniques de la réaction sulfatique interne du béton, $\mathrm{PhD}$ thesis, Université Paris Est, Paris.

Martin R.-P. \& Toutlemonde F. (2010) Design of a heat treatment representative of the curing conditions of a massive concrete structure. Bulletin des Laboratoires des Ponts et Chaussées 278: 49-63.

Martin R.-P., Bazin C., Billo J., Estivin M., Renaud J.-C., Toutlemonde F. (2012) Experimental evidence for understanding DEF sensitivity to early-age thermal history. International workshop on crack control of mass concrete and related issues concerning early-age of concrete structures Concrack3, Paris, France: 45-54.

Pourchez J., Valdivieso F., Grosseau P., Guyonner R., Guilhot B.. (2006) Kinetic modelling of the thermal decomposition of ettringite into metaettringite. Cement and Concrete Research 36: 20542060.

Ramlochan T., Zacarias P., Thomas M.D.A., Hooton R.D. (2003) The effect of pozzolans and slag on the expansion of mortars cured at elevated temperature - Part I: Expansive behaviour. Cement and Concrete Research 33: 807-814.

Seignol J.-F., Baghdadi N., Toutlemonde, F. (2009) A macroscopic chemo-mechanical model aimed at re-assessment of DEF affected concrete structures. 1st International conference on computational technologies in concrete structures CTCS'09, Jeju, Korea: 442-440.

Scrivener K.L., Damidot D., Famy C. (1999) Possible mechanisms of expansion of concrete exposed to elevated temperatures during curing (also known as DEF) and implications for avoidance of field problems. Cement, Concrete and Aggregates 21(1): 93-101.

Tailhan J.-L., D'Aloïa L., Autuori P. (2010) Numerical simulations of the early-age behavior of concrete structures: modeling and feedback. Bulletin des Laboratoires des Ponts et Chaussées 278: 64-77.

Taylor H.F.W., Famy C., Scrivener K.L. (2001) Delayed Ettringite Formation. Cement and Concrete Research 26(2): 683-693.

Waller V. (2000) Relations entre composition des bétons, exothermie en cours de prise et résistance en compression, ERLPC, OA 35. Laboratoire Central des Ponts et Chaussées, Paris. 